\title{
Recent Technology of Clinical Microbiology; Whole Genome Sequencing (WGS) and Matrix-Assisted, Laser Desorption Ionization-Time of Flight Mass Spectrometry (MALDI-TOF MS)
}

Jeongsook $\mathbf{Y}^{*}$

Kangnam Koryo Hospital, Seoul, Korea

\begin{abstract}
The hottest issue in the field of clinical microbiology in latest years is the application of whole genome sequencing (WGS) to the clinical diagnostics and therapeutics through the methodology of Next generation sequencing. The application of WGS includes species identification, epidemiological study and study of antimicrobial resistance and so on. The single nucleotide polymorphism (SNP) based analysis increases the accuracy and sensitivity of species identification by marked discriminatory power, and the ability of tracking of transmission process enables infection control including outbreaks. We can clarify the antimicrobial resistance mechanism of extended spectrum betalactamases or carbapenemases and elucidate the mechanism of horizontal transfer of mobile genomic islands. In case of setting up a new PCR method, the target and the primer could be chosen by using WGS databases.

The MALDI-TOF method is used in nearly all laboratories, identifying bacterial, fungal and mycobacterial species. Direct identification in blood culture bottle is possible in bacteremia patients, and Beta-lactamases or Carbapenemases can be detected. In addition, it can be applied in Shiga toxin E. coli, Salmonella serotypes or $C$. difficile ribotypes.
\end{abstract}

Keywords: Clinical microbiology; Whole genome sequencing; MALDI-TOF MS

\section{Introduction}

The most important issue in clinical microbiology is the accurate and timely diagnosis. Molecular diagnoses have markedly improved pathogen detection and identification. The most recent development in this area is the application of whole-genome sequencing (WGS).

Following the recent introduction of next-generation sequencing to clinical medicine, the application of whole-genome sequencing (WGS) to microbiological diagnostics and therapeutics has become the hottest issue in the field of clinical microbiology [1]. WGS involves species identification, epidemiological studies and antimicrobial resistance studies [2]. The use of single-nucleotide polymorphism (SNP)-based analysis in WGS increases discriminatory power in advanced research, as well as in clinical diagnostics and therapeutics.

Phylogenetic analysis using 16S ribosomal RNA (16S rRNA) sequencing is a gold standard for the identification of species and subspecies. However, the technique cannot differentiate between closely related strains such as those found in the genus Neisseria; this issue can be resolved using WGS. A great number of species and subspecies are now represented in the WGS database, which is updated continuously. Occasionally, WGS reveals that a bacterial species or subspecies has been misidentified by phenotyping, resulting in a taxonomic change [3].

Another significant application of WGS is in the field of antimicrobial resistance. Resistome analysis, analysis of horizontal transfer of mobile genomic islands, and recombination analysis greatly assist the development of antimicrobial therapeutics, particularly in the context of multidrug-resistant (MDR) strains containing extended-spectrum betalactamases (ESBLs) or carbapenemases [4]. Moreover, transmission tracking is enabled by phylogenetic mapping, and the analysis of clonality or lineage support infection surveillance and control $[5,6]$.

Mass spectrometric techniques for determination of singlecomponent have developed greatly over the past decade. According to ion source, mass spectrometry include Multimode ionization, Nano electrospray ionization, Matrix assisted laser desorption ionization, Electronic ionization and Chemical ionization etc. According to mass spectrometry filter, mass spectrometry includes Quadrupole, Sector, Time of flight, FT-ICR, Ion Trap, Orbitrap.

Matrix assisted laser desorption ionization time of flight mass spectrometry (MALDI-TOF) is a standard in mass spectrometry in general and protein analysis in particular. Its advantage includes high sensitivity, tolerance to buffers, fast data acquisition, and simple and robust instrumentation. MALDI-TOF was first used for bacterial identification 10 years ago, and nearly all identification in North America and Europe is now carried out using the technique $[7,8]$.

Setting up bioinformatics software can be costly and timeconsuming, and complicated computing infrastructure is a major limitation. However, once it is set up, MALDI-TOF can be more efficient in terms of cost and workload [9]. Philippe et al. reported that they had used MALDI-TOF for direct identification in blood culture vials, which shortened the procedure [10]. Moreover, beta-lactamases or carbapenemases can be detected using MALDI-TOF [11,12].

\section{Materials and Methods}

\section{WGS methods}

The genomic DNA was extracted from isolates (Qiagen, Hilden,

*Corresponding author: Jeongsook Y, Kangnam Koryo Hospital, Seoul, Korea, Tel: 8201090551345; E-mail: js1345@medigate.net, js13455844@hanmail.net

Received June 06, 2016; Accepted June 19, 2017; Published June 26, 2017

Citation: Jeongsook Y (2017) Recent Technology of Clinical Microbiology; Whole Genome Sequencing (WGS) and Matrix-Assisted, Laser Desorption IonizationTime of Flight Mass Spectrometry (MALDI-TOF MS). J Microb Biochem Technol 9:138-142. doi: 10.4172/1948-5948.1000357

Copyright: (c) 2017 Jeongsook Y. This is an open-access article distributed under the terms of the Creative Commons Attribution License, which permits unrestricted use, distribution, and reproduction in any medium, provided the original author and source are credited. 
Germany) and whole-genome Illumina sequencing was carried out using a MiSequence 2000 system (Illumina Inc., San Diego, CA, USA). Illumina, 454 GS Junior System (Roche), Ion Torrent Personal Genome Machine (PGM) (Life Technologies) and PacBio RS (Pacific Biosciences) are certified trademarks [13].

Processing is required before analysis owing to the unassembled short read sequence. Trimmed, filtered sequencing reads are mapped to the genome of the reference strain for SNP analysis [1]. The target insert size and paired end reads are determined and assembled using bioinformatics tools for phylogenetic and population structure [14]. Which is added that para have to come after $[15,16]$.

\section{Methods of MALDI-TOF}

In an MS analysis utilizing MALDI as a ionization mechanism, a saturated solution of a low-mass organic compound, called a matrix, is added to the sample, and the mixture is then spotted onto a metal target plate for analysis. Upon drying, the clinical material and the matrix cocrystallize and form a solid deposit. This sample-matrix crystal, present on the surface of the metal plate, is irradiated by using a UV laser beam. Pulsation by the laser provokes both the matrix and clinical sample to rapidly sublimate from the solid phase into the gas phase, forming a plume containing ions from both the matrix and the clinical sample. Once ionized, proteins within the clinical specimen are analyzed by a component of the mass spectrometer called the mass analyzer to reveal characteristic information about the composition of the sample in the context of a spectrum of mass-to-charge $(\mathrm{m} / z)$ ratios.

In bacterial identification, single colonies are suspended in $70 \%$ ethanol, vortexed, and concentrated by centrifugation. The supernatant is discarded and the cells are resuspended in $50 \mathrm{uL}$ of $70 \%$ formic acid, an equal volume of acetonitrile is added, vortexed, concentrated by centrifugation. One $\mathrm{uL}$ of the supernatant is spotted on the target plate, allowed to evaporate to dryness, and overlaid with the matrix of a-cyano-4-hydroxy-cinnamic acid. If the solution contains a minimum of 5-10 × $10^{6}$ cells/uL, sufficient spectra are obtained for identification.

\section{Results and Discussion}

\section{Application of WGS}

Mechanism of communication of asymptomatic carriers and pathogens: The respiratory and gastrointestinal tracts permit colonization of asymptomatic carriers such as Neisseria spp. in the respiratory tract and Clostridium difficile in the gastrointestinal tract $[17,18]$. C. difficile, the most serious source of hospital-associated infections, is categorized as an asymptomatic carrier and causes diseases when normal neighboring bacteria are suppressed by antimicrobial drugs. An avirulent strain that has colonized the gastrointestinal tract can acquire the gene that encodes toxin $\mathrm{b}(\mathrm{tcdB})$ or an antimicrobialresistant gene from the same species or from a different species that is present nearby [19]. Streptococci or Neisseria species colonizing the respiratory tract can acquire specific virulence genes or cephalosporinresistant genes which are inserted into specific plasmids, prophages or mobile genomic islands [22]. WGS can be used to elucidate such a mechanism [20].

In addition, a specific sequence type (ST) of the asymptomatic carrier is associated with a specific disease. For example, ST131 is thought to be a disease-causing sequence type in diarrheagenic Escherichia coli [21]. And Clair et al. suggested that a specific ST is associated with cephalosporin-resistant Neisseria gonorrhoeae [22].

WGS supports sequence types and genetic information on MDR strains.
Species identification: A large number of bacterial species and subspecies have been identified by WGS, and the results have been added to the WGS databases, BIGSdb $[23,24]$. SNP-based analysis increases the accuracy of species and subspecies identification $[3,25]$. Phenotyping my lead to the misidentification of species or subspecies, as revealed by the results of WGS, leading to taxonomy changes [3]. For example, Staphylococcus aureus has been misidentified by phenotyping and subsequently the identification has been changed to Staphylococcus argenteus or Staphylococcus schweitzeri by WGS [25].

Epidemiology study: Multi-locus sequence typing (MLST) was developed by Maiden in 1998 and replaced pulsed-field gel electrophoresis (PFGE), which was the gold standard of strain typing at the time. MLST analyzes seven housekeeping genes by sequencing; it determines the sequence type and has high discriminatory power. However, it is limited by its inability to differentiate between closely related strains. WGS has greater discriminatory power because it is based on SNP analysis. It is possible to determine bacterial origin, evolution, clonality and lineage by constructing a phylogenetic map [26,27].

Ribosomal MLST (rMLST), whole-genome MLST (wgMLST) and core-genome MLST (cgMLST) can be carried out using bioinformatic tools for epidemiology studies. The cgMLST approach uses in silico analysis which analyzes more than dozens of housekeeping genes, and is an SNP-based technique, which results in higher discriminatory power and easier standardization. SeqSphere+ bioinformatic software (Ridom $\mathrm{GmbH}$ ) has been used for cgMLST [5]. In outbreak cases, the specific ST can be determined through wgMLST, cgMLST or rMLST and rare STs can be detected owing to the high discriminatory power of the SNP assay. For example, it was possible to control an outbreak by Neisseria meningitides by detecting ST6697, a rare disease-causing ST $[14,28]$. When applied to infection control, it is possible to construct a minimum span tree and to track a strain by excluding the strain from transmission if it has a greater distance than that of the threshold allele [6]

Study of antimicrobial resistance: A specific antimicrobial resistance gene can be detected by real-time polymerase chain reaction (RT-PCR), microarray assay or fluorescence in situ hybridization (FISH) and beta-lactamases or carbapenemases have been detected using MALDI-TOF [11,12]. In MDR species such as MRSA, VRE, MDR Enterobacteriaceae, MDR Pseudomonas and Acinetobacter, WGS detects an antimicrobial resistome such as ESBLs or carbapenemases by SNP-level analysis, contributing to antimicrobial therapy. The structures of ESBL such as CTX-M and carbapenemase such as KPC or NDM-1 have been elucidated by WGS analysis and the presence of mobilized colistin resistance gene $\mathrm{mcrl}$, which has become the focus of much attention in recent years, can be confirmed by WGS $[4,29]$. We have made progress in infection control and surveillance by observing the horizontal transfer of plasmids or mobile genetic elements, and by analyzing mutation or recombination events. The existence of mobile prophages in Staphylococcus lugdunensis has been confirmed using WGS and bioinformatics tools, which have discovered genetic recombination [30].

Yamamoto et al. used WGS to detect a plasmid that included the blaIMP19 gene in both Achromobacter xylosoxidans and Klebsiella pneumoniae, suggesting that plasmid-borne blaIMP19 is horizontally transferred from A. xylosoxidans to K. pneumoniae [31]. Willemsen et al. suggested the horizontal transfer between patients by detecting a similar Inc L/M plasmid in both E. coli and K. pneumoniae strains in each patient [32]. 
There has also been a report of reduced susceptibility to ciprofloxacin in Salmonella typhi and the spread of this easily transferable strain containing an antimicrobial resistome which is inserted into mobile genomic elements causes significant results [33]. For example, it was found that MDR S. typhi, which occurred in Zambia between 2010 and 2012, contained the MDR plasmid IncHIl derived from the transformed H58B haplotype, illustrating the advantage of WGS [34].

K. pneumoniae is one of the most important causes of healthcareassociated infection. Easily transmissible strains of the bacterium such as ST258, which resides in some colonies and acquires virulent and antimicrobial-resistant genes, can be transmitted unobtrusively, causing outbreaks of the MDR strain [35]. WGS is required for infection control in such situations.

Using WGS, Mellmann et al. developed a surveillance program that controls prospective ESBL strains such as CTX-M, carbapenemase strains such as KPC, NDM, or OXA, plasmid-mediated quinoloneresistant strains, aminoglycoside-resistant strains and colistin-resistant mor1 strains in K. pneumoniae [6].

Detection of a virulence gene: Mei et al. used by WGS to detect K2aerobactin-kfu-rmpA virulence factor in K. pneumoniae, and there is a report that hypervirulent genes wzi, pk2044_00025, and pk2044_00325 have been found in K. pneumonia [36,37].

Infection control: In the case of hospital-associated infections, calculating the genetic distance of MDR strains that can follow the transmission route facilitates the control and surveillance of infection [38]. Mellmann et al. constructed a cgMLST that enables transmission tracking by determining the genetic distance using an SNP-based method in MDR strains such as MRSA, VRE, MDR E. coli, MDR K. pneumonia and MDR Pseudomonas [6].

Therefore, a program can be constructed for prospective infection control in a clinical setting.

Vaccine replacement strain: Following advances in vaccine development, vaccines conjugated to capsular polysaccharides have reduced morbidity and mortality caused by $S$. pneumoniae infection by $90 \%$ over the past decade. The vaccine's ability to prevent diseases is remarkable in infections of bacterial species such as $N$. meningitides and Bordetella pertussis, and viruses such as measles, Ebola and Zika.

However, owing to the effect of selective pressure following vaccination, non-vaccine replacement strains can become predominant and serotype or sequence type switch or transformation is possible, leading to the development of new MDR clones [38]. WGS can be used to determine whether a serotype or sequence type has been switched or transformed and to detect the development of new MDR clones [39]. Following vaccination, it is now possible to determine whether a fever is due to a vaccine strain or to a new wild-type infection [40]. The elucidation of genomic structure and the monitoring of serotype or sequence type switch or transformation are very useful for the design and development of new vaccines.

Primer design and setting up new PCR assays: WGS databases (e.g. http://www.mlst.net, http://pubmlst.org) can be used to develop new PCR assays, which require a target sequence and the design of a primer [41,42]. For example, real-time PCR can be used to differentiate between Campylobacter jejuni and Campylobacter coli. Best et al. reevaluated this method by analyzing more than 1,700 Campylobacter genomes extracted from the pubmlst database [41]. The primer and probe sequences of mapA and $c e u E$, which are PCR targets for $C$. jejuni and C. coli, were analyzed in silico. As a result, a real-time PCR assay identified $99.7 \%$ of the isolates accurately. However, the reduced specificity of $C$. coli identification was determined to be due to introgression in mapA or sequence diversity in ceuE. This demonstrates how a WGS database can be used for re-evaluate previous PCR experiments and primers. Haemophilus haemolyticus is occasionally indistinguishable from non-typeable Haemophilus influenza, so with comparative genomic analysis, a new real-time PCR assay was developed that targets purT proving that a WGS database can be used to develop a new PCR or design a new primer [42].

\section{Application of MALDI-TOF}

Species identification: MALDI-TOF has completely replaced culture with biochemical methods for species identification in North America and Europe and large part in the remaining area of the world. VITEK MS (bioMérieux) and Biotyper (Bruker Daltonics) are examples of commercially available MALDI-TOF systems [7,8]. A recent report revealed that the MALDI-TOF assay had high correspondence with a biochemical culture and test (44), and MALDI-TOF and 16S rDNA analysis also had high correspondence, proving that MALDI-TOF is a rapid and accurate method [44,45]. Skin diphtheroids had been regarded as contaminants until MALDI-TOF enabled species identification, and reports of them acting as pathogens has increased [46].

MALDI-TOF reduces the identification time, but has limitations; it is subjective with regard to interpreting $\mathrm{m} / \mathrm{z}$ peaks, is prone to technical variations, and has difficulty in identifying rare species because the database is still being updated $[47,48]$.

Direct species identification in blood culture vials: Recently, MALDI-TOF has been applied directly to positive blood culture bottles for the rapid identification of pathogens, leading to reductions in turnaround time and potentially beneficial patient impacts. The development of a commercially available extraction kit (Bruker Sepsityper) for use with the Bruker MALDI BioTyper has facilitated the processing required for identification of pathogens directly from positive from blood cultures.

The authors of one study reported that the accuracy and correspondence with the VITEK 2 system were excellent, and it reduced the identification time when a species was identified directly in blood culture vials.

Species identification in $\mathbf{3} \mathbf{~ h}$ culture: This method is especially suitable for rapid identification of $E$. coli, the most common cause of urinary tract infections and urosepsis. Turnaround time for identification using U-si-MALDI-TOF (urine short incubation MALDI-TOF) compared with conventional urine culture was improved from $24 \mathrm{~h}$ to $3 \mathrm{~h}[49]$.

Detection of beta-lactamase and carbapenemase: ESBL or carbapenemase has been detected using MALDI-TOF by analyzing mass peak [11]. When observing another method for the detection of carbapenemase, isolates were incubated with ertapenem; the results were considered positive revealing carbapenem hydrolysis when the peak at $\mathrm{m} / \mathrm{z} 475$ disappeared completely $[12,50]$.

Additional applications: Virulent clone or sequence types and specific antimicrobial resistant types have been identified by confirming specific mass peaks. Furthermore, Shiga toxin E. coli, Salmonella serotypes, $C$. difficile ribotypes and Vibrio cholera phenotypes have also been identified [51-55]. 
Citation: Jeongsook Y (2017) Recent Technology of Clinical Microbiology; Whole Genome Sequencing (WGS) and Matrix-Assisted, Laser Desorption Ionization-Time of Flight Mass Spectrometry (MALDI-TOF MS). J Microb Biochem Technol 9:138-142. doi: 10.4172/1948-5948.1000357

\section{Conclusion}

This review has shown that WGS technology can be used for bacterial, viral and fungal identification and can also be applied to epidemiological studies including clinical outbreak and antimicrobial resistance studies. MALDI-TOF technology is used for species identification in nearly all laboratories, and has been used for direct identification in blood culture bottles and for the detection of betalactamases or carbapenemases. Specific phenotypes can also be detected.

\section{References}

1. Didelot X, Bowden R, Wilson DJ, Peto TEZ, Crook DW (2012) Transforming clinical microbiology with bacterial genome sequencing. Nat Rev Genet 13: 601-612.

2. Kwong JC, McCallum N, Sintchenko V, Howden BP (2015) Whole genome sequencing in clinical and public health microbiology. Pathology 47: 199-210.

3. Doijad S, Ghosh H, Glaeser S, Kämpfer P, Chakraborty T (2016) Taxonomic reassessment of the genus Elizabethkingia using whole genome sequencing Elizabethkingia endophytica kämpfer et al. 2015 is a later subjective synonym of Elizabethkingia anopheles kämpfer et al. 2011. Int J Syst Evol Microbiol 66 : 4555-4559.

4. Huang W, Wang G, Sebra R, Zhuge J, Yin C, et al. (2017) Emergence and evolution of multidrug-resistant Klebsiella pneumonia with both blaKPC and blaCTX-M integrated in chromosome. Antimicrob Agents Chemother pii: AAC.00076-17.

5. Chen Y, Gonzalez-Escalona N, Hammack TS, Allard MW, Strain EA, et al (2016) Core genome multi-locus sequence typing for identification of globally distributed clonal groups and differentiation of outbreak strains of listeria monocytogenes. Appl Environ Microbiol 82: 6258-6272.

6. Mellmann A, Bletz S, Böking T, Kipp F, Becker K, et al. (2016) Real-time genome sequencing of resistant bacteria provides precision infection control in an institutional setting. J Clin Microbiol 54: 2874-2881.

7. Rychert J, Creely D, Mayo-Smith LM, Calderwood SB, Ivers LC, et al. (2015) Evaluation of matrix-assisted laser desorption ionization-time of flight mass spectrometry for identification of Vibrio cholerae. J Clin Microbiol 53: 329-331.

8. Xiao D, Ye C, Zhang H, Kan B, Lu J, et al. (2014) The construction and evaluation of reference spectra for the identification of human pathogenic microorganisms by MALDI-TOF. PLOS ONE 9: e106312.

9. Croxatto A1, Prod'hom G, Greub G (2012) Applications of MALDI-TOF mass spectrometry in clinical diagnostic microbiology. FEMS Microbiol Rev 36: 380-407.

10. Lagacé-Wiens PRS, Adam HJ, Karlowsky JA, Nichol KA, Pang PF, et al. (2012) Identification of blood culture isolates directly from positive blood cultures by use of matrix-assisted laser desorption ionization - time of flight-mass spectrometry and a commercial extraction system: Analysis of performance, cost and turnaround time. J Clin Microbiol 50: 3324-3328.

11. Schaumann R, Knoop N, Genzel GH, Losensky K, Rosenkranz C, et al. (2012) A step towards the discrimination of beta-lactamase-producing clinical isolates of Enterobacteriaceae and Pseudomonas aeruginosa by MALDI-TOF mass spectrometry. Med Sci Monit 18: MT71-77.

12. Carvahaes CG, Cayo R, Visconde MF, Barone T, Frigatto EA, et al. (2014) Detection of carbapenemase activity directly from blood culture vials using MALDI-TOF MS; a quick answer for right decision. J Antimicrob Chemoth 69: 2132-2136.

13. Loman NJ, Misra RV, Dallman TJ, Constantinidou C, Gharbia SE, et al. (2012) Perfromance comparison of benchtop high-throughput sequencing platforms. Nat Biotechnol 30: 434-439.

14. Bratcher HB, Corton C, Jolley KA, Parkhill J, Maiden MCJ (2014) A geneby-gene population genomics platform: De novo assembly, annotation and genealogical analysis of 108 representative Neisseria meningitidis genomes. BMC Genomics 15: 1138-1153.

15. Francisco AP, Bugalho M, Ramirez M, Carriço JA (2009) Global optimal eBURST analysis of multilocus typing data using a graphic matroid approach BMC Bioinformatics 10: 152.
16. Stamatakis A, Ludwig T, Meier H (2005) RAxML-III: A fast program for maximum likelihood-based inference of large phylogenetic trees. Bioinformatics 21: 456.

17. Weyand NJ (2017) Neisseria models of infection and persistence in the upper respiratory tract. Pathog Dis 75 .

18. Furuya-Kanamori L, Marquess J, Yakob L, Riley TV, Paterson DL, et al. (2015) Asymptomatic Clostridium difficile colonization: Epidemiology and clinical implications. BMC Infect Dis 15: 516.

19. Brouer MS, Roberts AP, Hussain H, Williams RJ, Allan E, Mullany P (2013) A horizontal gene transfer converts non-toxigenic Clostridium difficile strains into toxin producers. Nat Commun 4: 2601.

20. Ochman H, Lawrence JG, Groisman EA (2000) Lateral gene transfer and the nature of bacterial innovation. Nature 405: 299-304.

21. Stephen JS, David JR, Jacob OK, Matthew WS, Bethany S, et al. (2015) Large scale genomic sequencing of extra intestinal pathogenic Escherichia coli strains. Genome Res 25: 119-128.

22. Claire C, Sylvain K, Maïté M, Nelly F, Guy LR, et al. (2016) Ceftriaxone resistant Neisseria gonorrhoeae isolates (2010 to 2014) in France characterized by using whole-genome sequencing. Antimicrob Agents Chemother 60: 6962-6964.

23. Cowley LA, Beckett SJ, Chase-Topping M, Perry N, Dallman TJ, et al. (2015) Analysis of whole genome sequencing for the Escherichia coli O157:H7 typing phages. BMC Genomics 16: 271

24. Harris SR, Cartwright EJP, Torok ME, Holden MTG, Brown NM, et al. (2013) Whole-genome sequencing for analysis of an outbreak of methicillin-resistant Staphylococcus aureus: A descriptive study. Lancet Infect Dis 13: 130-136.

25. Steven YCT, Frieder S, Matthew JE, Jukka C, Bruno P, et al. (2015) Nove Staphylococcal species that form part of pigmented Staphylococcus argenteus $\mathrm{sp}$. Nov. and the non-human primate-associated Staphylococcus schweizen sp. Nov. Int J Syst Evol Microbiol 65: 15-22.

26. Sarovich DS, Price EP (20140 SPANDx: A genomics pipeline for comparative analysis of large haploid whole genome. Re-sequencing datasets. BMC Res Notes 7: 618.

27. DePristo MA, Banks E, Poplin R, Garimella KV, Maquire JR, et al. (2011) A framework for variation discovery and genotyping using next-generation DNA sequencing data. Nat Genet 43: 491-498.

28. Robert MM, Carina B, Lois O'C, Kenneth M, Keith AJ, et al. (2016) Resolution of a protracted serogroup $b$ meningococcal outbreak with whole-genome sequencing shows interspecies genetic transfer. J Clin Microbiol 54: 2891 2899.

29. Schürch AC, van Schaik W (2017) Challenges and opportunities for wholegenome sequencing-based surveillance of antibiotic resistance. Ann N Y Acad Sci 1388: 108-120.

30. Argemi X, Martin V, Loux V, Dahyot S, Lebeurre J, et al. (2017) Whole genome sequencing of 7 strains of Staphylococcus lugdunensis allows identification of mobile genetic elements. Genome Biol: 9.

31. Yamamoto M, Matsumura Y, Gomi R, Matsuda T, Tanaka M, et al. (2016) Interspecies dissemination of mobilizable plasmid harboring blalMP-19 and the possibility of horizontal gene transfer in a single patient. Antimicrob Agents Chemother 60: 5412-5419.

32. Willemsen I, Van Esser J, Kluytmans M, Zhou K, Rossen JW, et al. (2016) Retrospective identification of a previously undetected clinical case of oxa-48producing K. pneumoniae and E. coli: The importance of adequate detection guidelines. Infection 44: 107-110

33. Duy PT, Abhilasha K, Sabina D, Nhan HT, Corinne NT, (2016) A novel ciprofloxacin-resistant sub clade of H58 Salomonella typhi is associated with fluoroquinolone treatment failure. eLife: e14003.

34. Hendriksen RS, Leekicharoenphon $P$, Lukjancenko $O$, Lukwesa-Musyan C, Tambatamba B, et al. (2015) Genomic signature of multidrug-resistant Salmonella enterica serovar typhi isolates related to a massive outbreak in Zambia between 2010 and 2012. J Clin Microbiol 53: 262-272.

35. Bowers JR, Kitchel B, Driebe EM, MacCannell DR, Roe C, et al. (2015) Genomic analysis of the emergence and rapid global dissemination of the clonal group 258 Klebsiella pneumoniae pandemic. PloS ONE 10: e0133727.

36. Mei YF, Liu PP, Wan LG, Liu Y, Wang LH, et al. (2017) Virulence and genomic 
Citation: Jeongsook Y (2017) Recent Technology of Clinical Microbiology; Whole Genome Sequencing (WGS) and Matrix-Assisted, Laser Desorption Ionization-Time of Flight Mass Spectrometry (MALDI-TOF MS). J Microb Biochem Technol 9:138-142. doi: 10.4172/1948-5948.1000357

feature of a virulent Klebsiella pneumoniae sequence type 14 strain of serotype K2 harboring blaNDM5 in China. Front Microbiol 23: 335.

37. Keh-Ming W, Ling-Hui L, Jing-Jou Y, Nina T, Tsai-Lien L, et al. (2009) Genome sequencing and comparative analysis of Klebsiella pneumonia NTUH-K2044, a strain causing liver abscess and meningitis. J Bacteriol 191: 4492-4501.

38. Dallman TJ, Byrne L, Ashton PM, Cowley LA, Perry NT, et al (2015) Wholegenome-sequencing for national surveillance of shiga toxin-producing Escherichia coli 0157. Clin infect Dis 61: 305-312.

39. Makarewicz O, Lucas M, Brandt C, Herrmann L, Albersmeier A, et al. (2017) Whole genome sequencing of 39 invasive Streptococcus pneumonia sequence type 199 isolates revealed switches from serotype 19A to 15B. PLOS ONE 12: e0169370.

40. Bankamp B, Takeda M, Zhang Y, Xu W, Rota PA (2011) Genetic characterization of measles vaccine strains. J Infect Dis 204 Suppl 1: S533-548.

41. Jansen van Rensburg MJ, Swift C, Cody AJ, Jenkins C, Maiden MC (2016) Exploiting bacterial whole-genome sequencing data for evaluation of diagnostic assays: Campylobacter species identification as a case study. J Clin Microbio 54: $2882-2890$.

42. Hu F, Rishishwar L, Sivadas A, Mitchell GJ, Jordan K, et al. (2016) Comparative genomic analysis of Haemophilus haemolyticus and non-typeable Hemophilus influenzae and a new testing scheme for their discrimination. J Clin Microbio 54:3010-3017.

43. Patrick RM (2012) What Is new in clinical microbiology-microbial identification by MALDI-TOF mass spectrometry. J Mol Diagn. 14: 419-423.

44. Febbraro F, Rodio DM, Puggioni G, Antonelli G, Pietropaolo V, et al. (2016) MALDI-TOF MS versus vitek ${ }^{\circledR} 2$ : Comparison of systems for the identification of microorganisms responsible for bacteremia. Curr Microbiol 73: 843-850.

45. Eigner U, Holfelder M, Oberdorfer K, Betz-Wild U, Bertsch D, et al. (2009) Performance of a matrix-assisted laser desorption ionization-time-of-fligh mass spectrometry system for the identification of bacterial isolates in the clinical routine laboratory. Clin Lab 55: 289-96.

46. Nagy E, Urban E, Becker S, Kostrzewa M, Vörös A, et al. (2013) MALDI-TOF
MS fingerprinting facilitates rapid discrimination of phylotypes I, II and III of Propionibacterium acnes. Anaerobe 20: 20-26.

47. Agergaard CN, Knudsen E, Dargis R, Nielsen XC, Christensen JJ, et al (2017) Species identification of Streptococcus bovis group isolates causing bacteremia; a comparison of two MALDI-TOF MS systems. Drug Microbiol Infect Dis 88: 23-25.

48. Wattal C, Oberoi JK, Goel N, Raveendran R, Khanna S (2017) Matrix-assisted laser desorption ionization time of flight mass spectrometry (MALDI-TOF MS) for rapid identification of microorganisms in the routine clinical microbiology. Eur J Clin Microbiol Infect Dis 36: 807-812.

49. Haiko J, Savolainen LE, Hilla R, Pätäri-Sampo A (2016) Identification of urinary tract pathogens after $3 \mathrm{~h}$ urine culture by MALDI-TOF mass spectrometry. $J$ Microbiol Methods 129: 81-84.

50. Lartigue MF, Kostrzewa M, Salloum M, Haguenoer E, Hery-Arnaud G, et a (2011) Rapid detection of "highly virulent" groupB Streptococcus ST-17 and emerging ST-1 clones by MALDI-TOF mass spectrometry. J Microbiol Methods 86: 262-265.

51. Wolters M, Rohde H, Maier T, Belmar-campos C, Franke G, et al. (2011) MALDI-TOF MS fingerprinting allows for discrimination of major methicillinresistant Staphylococcus aureus lineages. Int J Med Microbiol 301: 64-68.

52. Fagerquist CK, Zaragoza WJ, Sultan O, Woo N, Quinones B, et al. (2014) Top-down proteomic identification of Shiga toxin 2 subtypes from shiga toxinproducing Escherichia coli by matrix-assisted laser desorption ionizationtandem time of flight mass spectrometry. Appl Environ Microbiol 10:1128.

53. Kuhns M, Zautner AE, Rabsch W, Zimmernann O, Weig M, et al. (2012) Rapid discrimination of Salmonella enterica serovar typhi from other serovars by MALDI-TOF mass spectrometry. PLoS ONE 7: e40004

54. Reil M, Erhard M, Kuijper EJ, Kist M, Zaiss H, et al. (2011) Recognition of Clostridium difficile PCR-ribotypes 001, 027 and 126/078 using an extended MALDI-TOF MS system. Eur J Clin Microbiol Infect Dis 30: 1431-1436.

55. Schirmeister F, Dieckmann R, Bechlars S, Bier N, Faruque SM, et al. (2014) Genetic and phenotypic analysis of Vibrio cholerae non-O1, non-O139 isolated from German and Austrian patients. Eur J Clin Microniol Infect Dis 33: 767-778. 